\title{
DISEÑO PEDAGÓGICO VIRTUAL DE DESARROLLO EMPRESARIAL CON APOYO DE LAS TIC
}

\section{VIRTUAL PEDAGOGICAL DESIGN FOR BUSINESS DEVELOPMENT WITH ICT SUPPORT}

\author{
William Ricardo Zambrano \\ Doris Emilia Guerrero²
}

\section{RESUMEN}

Se presenta el estado del arte y las proyecciones sobre modelos pedagógicos virtuales de desarrollo empresarial en el mundo, a través de una revisión histórica, documental y bibliográfica de experiencias emblemáticas de universidades que los han implementado. En la primera parte, se analizan los modelos e-learning de universidades de Europa, América y Colombia, basados en la incorporación de las Tecnologías de Información y Comunicación (TIC) y metodologías utilizadas para optimizar el aprendizaje. En la segunda, se articulan los hallazgos, a fin de propiciar ideas innovadoras para el diseño pedagógico virtual de desarrollo empresarial en línea. La tercera, valida el modelo en dos cursos académicos. El estudio, deja como conclusión, que a pesar que existen diferentes modelos pedagógicos de desarrollo empresarial virtual, se requiere la creación de otros que permitan, con apoyo de las TIC, alcanzar mayores competencias, cooperación y conectividad, a través de plataformas y de mega redes de aprendizaje, que faciliten la construcción de nuevo conocimiento compartido, como aporte a la universidad en general $y$, en particular a la colombiana, en el desarrollo de su contexto inmediato frente a la globalización de la educación y de la empresa.

${ }^{1}$ Comunicador Social - Periodista. M.Sc. Comunicación, Docente Investigador Ingeniería Comercial. U.D.C.A. e-mail: zambrano_william@hotmail.com

2 Abogada. Filósofa. M.Sc. Estudios Humanísticos. Docente Investigadora. Ingeniería Comercial U.D.C.A. e-mail: dorisgue@udca.edu.co
Palabras clave: Sociedad del conocimiento, tecnologías de la información y comunicación, educación virtual, modelos pedagógicos.

\section{SUMMARY}

The state of the art and projections related to virtual pedagogical business models developed in the world is presented through a historical, documentary and bibliographical review of emblematic experiences by universities that have implemented these models. In the first part, the models of e-learning in European, American and Colombian universities are analyzed, based on the incorporation of information and communication technologies (ICT) and methodologies used to optimize learning. In the second, the findings are articulated in order to promote innovative ideas for designing an educational virtual online business development. The third component validates the model in two academic years. The study leaves as conclusion that despite the existence of different models of educational virtual business development, the creation of others, with the support of ICT is required, to achieve greater competition, cooperation and connectivity across platforms and mega learning networks. This, to facilitate the construction of new, shared knowledge as contribution to the university in general and, in particular, to the Colombian one, in the development of its immediate context, in front of the globalization of education and commerce.

Key words: Society of knowledge, information technology and communication, virtual education, pedagogical models. 


\section{INTRODUCCIÓN}

Las actuales generaciones enfrentan cambios en diferentes ámbitos: científico, tecnológico, político, económico, social y cultural, "estas tendencias presentan desafíos para los sistemas educativos en cuanto a la selección de metodologías de enseñanza y aprendizaje virtual y las Tecnologías de Información y Comunicación (TIC) que se ofrecen a los discentes" (Denis, 1998). Además, el contexto empresarial se ha transformado en los últimos años (Bednar et al. 2007), lo que se evidencia en el entorno organizacional, dando lugar a procesos de democratización, a la incorporación de nuevos competidores, "a la integración en algunos sectores, a la desintegración en otros, a la profusión de información y comunicación, fusiones y alianzas, al surgimiento de bloques comerciales identificados" (Yarzábal, 2004), mayores demandas de calidad, servicio, a grandes avances tecnológicos de la mano de las TIC (Saettler, 2005), a la apertura del comercio internacional y a la creación global de nuevas estructuras de empresas.

Según Área (2005), "en la educación, la tecnología es y será un gran bastión en su desarrollo, apoyo a la formación del individuo, contenidos electrónicos, internet; elementos estos que enriquecen y ayudan a los procesos empresariales". La Sociedad de la Información y el Conocimiento (SIC) constituye un cambio, un nuevo modelo y una realidad que necesita liderazgo tecnológico para su desarrollo en los ámbitos empresariales. Su impacto en la sociedad global y sus repercusiones en la gestión empresarial, se ve reflejado en los cuestionamientos que se han generado sobre la forma en que los alumnos, en general y, en particular, los de la Facultad de Ingeniería Comercial de la Universidad de Ciencias Aplicadas y Ambientales U.D.C.A, aprenden y desarrollan sus competencias en la creación de entornos más efectivos, centrados en su futura actividad empresarial.

"Estos cambios responden a diversos factores, entre los cuales, se resalta el papel del conocimiento en la transformación empresarial" (Guerrero et al. 2007). Las Instituciones de Educación Superior (IES) y los empresarios están convencidos que el proceso pedagógico virtual de desarrollo empresarial tendrá un papel importante en la sociedad basada en la información: las TIC son el componente básico de la educación virtual y su manejo supone un esquema con base en su buen uso y eficiencia (Suengas et al. 2003).

Debido a estas transformaciones que afectan a las empresas y a las IES, se hace necesario implementar nuevos procesos pedagógicos virtuales de desarrollo empresarial dirigido a estudiantes de educación superior, con nuevas estrategias organizacionales, modernos enfoques didácticos y "metodológicos para formar líderes capaces de enfrentar los retos del presente siglo" (EDUTEKA, 2008).

Dentro de este ámbito emerge, de manera rápida y creciente en la educación, la virtualidad, la cual, hace referencia a los instrumentos y a los procesos utilizados para transmitir, producir, intercambiar información y conocimiento por medios electrónicos (Fundación Gabriel Piedrahita Uribe, 2007). Esta modalidad educativa, denominada e-learning, se centra en el proceso de enseñanza-aprendizaje apoyado por TIC, a través del medio virtual, que facilita la interactividad (Tinker, 2008).

En consecuencia, dadas las exigencias actuales en la educación, se propone evaluar las experiencias en modelos pedagógicos virtuales en países líderes en este tipo de formación, por lo cual, el presente estudio pretende dar respuesta a los siguientes interrogantes: ¿Cuáles son los nuevos enfoques educativos para crear un diseño de formación pedagógica en gestión y desarrollo empresarial virtual?, ćcómo aplicar el proceso pedagógico en línea para la cualificación del futuro empresario de la Facultad de Ingeniería Comercial? y ¿cuál es el nuevo papel del docente y discente en la educación virtual empresarial?

De otra parte, esta propuesta será importante para desarrollar asignaturas asistidas por tecnologías relacionadas con la empresa en los procesos de aprendizaje y que se pueden implementar a otros campos afines. El propósito de esta aplicación es la de mejorar el ejercicio académico, la formación docentediscente, las estrategias metodológicas empleadas en la gestión y desarrollo empresarial, con miras a favorecer la interactividad. 


\section{MATERIALES Y MÉTODOS}

Para adelantar la primera parte del estudio, se realizó un estado del arte sobre modelos pedagógicos virtuales de desarrollo empresarial con apoyo de las TIC, entre los periodos de 1990 a 2008, para evaluar sus debilidades y sus fortalezas e identificar diferentes enfoques educativos y tecnológicos. Se recurrió a bibliografía, mediante información de libros, de trabajos científicos, de documentos inéditos, de ponencias, de conferencias, de investigaciones previas, de revistas indexadas y de documentación pertinente en Internet.

Para cumplir con lo anterior, se tomó una muestra de $146(69,2 \%)$ experiencias significativas de instituciones de: Europa, 57 (39\%); Norteamérica, 18 (12.4\%); Latinoamérica, 48 (33\%) y Colombia, 23 (15.6\%), según los registros de acreditación entregados por Ministerios de Educación de cada país. Los criterios de selección de la muestra referenciada fueron los siguientes: universidades con mayor trayectoria internacional en la implementación de modelos pedagógicos virtuales, metodologías que desarrollan competencias empresariales e Instituciones que arrojan logros positivos en los procesos de aprendizaje en línea. Como resultado, se logró una representación de $72 \%$ de encuestas respondidas, lo que facilitó clasificar la información y complementarla a través de cada portal.

Para la segunda parte, se desarrolló una investigación teórica de tipo exploratorio, lo que permitió determinar los referentes empíricos y diseñar las estrategias de recolección de información y de análisis documental. La información, se recogió de los cursos académicos del programa de Ingeniería Comercial de la U.D.C.A: Legislación Comercial, en el que se identifican los requisitos legales para la creación, funcionamiento de las unidades de negocio y Comunicación Oral y Escrita, en el que se fundamentan procesos de interacción para el desarrollo óptimo de la comunicación organizacional.

Para el cumplimiento del anterior propósito, se aplicó para el levantamiento de datos técnicas de encuesta y de entrevista en profundidad, con el propósito de validar el diseño pedagógico virtual de desarrollo empresarial con apoyo de las TIC. Se tomó una muestra de 40 alumnos de los cursos mencionados, con el fin de conocer los aspectos considerados de mayor interés en el impacto y validación del diseño durante el proceso de aprendizaje. Para tal fin, no se consideraron variables como la edad, el nivel educativo y socioeconómico de los estudiantes, debido a que el currículo es flexible y no se exige para la inscripción de los cursos académicos ningún requisito.

Se presentaron dificultades en la identificación de las universidades que emplean, en su diseño pedagógico virtual, el desarrollo empresarial, empleando las TIC, como: algunas instituciones no diligenciaron las encuestas proporcionadas, otras no las hicieron llegar oportunamente. No obstante, los hallazgos permitieron conocer diagnósticos significativos de formatos a distancia, que proporcionaron un estudio sobre la mejor manera de favorecer los aprendizajes y la adquisición de nuevos conocimientos.

\section{RESULTADOS Y DISCUSIÓN}

Se obtuvo como resultado, la identificación de debilidades y fortalezas de los modelos pedagógicos virtuales nacionales e internacionales de desarrollo empresarial, lo que arrojó el siguiente análisis:

En Europa: la Universidad Oberta de Cataluña de España (UOC, 2008) es pionera en esta modalidad y su objetivo es satisfacer las necesidades de aprendizaje de los estudiantes, con el empleo intensivo de las TIC, para desarrollar empresa. De igual manera, las universidades de Málaga, Coruña, Vigo, del País Vasco, Politécnica de Madrid, Complutense, Santiago de Compostela, Sevilla, Cantabria, Les Illes Ballears, Centros tecnológicos andaluces, Murcia, Granada y Autónoma de Barcelona, implementan un modelo pedagógico de creación de empresa, a través de una metodología práctica: formulación de actividades, evaluación de respuestas a los ejercicios planeados mediante retroalimentación.

En Inglaterra, se destaca American International University in London, Universidad Sheffield, Universidad London, Bournemouth, Brunel, Uxbridge, City University London y University of North London, las cuales, enfatizan aprendizaje significativo y socialmente pertinente, mediante interacción individual o grupal entre alumnos, equipo pedagógico y contenidos.

En Francia, la Central European University, University of Jewish Studies y en Hungría, Kodolanyi Janos University College, se apoyan en el Modelo Concord (CC, 2008), 
centrado en la disponibilidad de comunicación vía Internet, siguiendo un calendario de actividades, a través de evaluación permanente, según Monereo (2007): "Pasaron del concepto de aprendizaje basado en la adquisición de conocimiento al de su construcción".

En América: se ha desarrollado lentamente la educación virtual empresarial en comparación con Europa; sin embargo, hay universidades que están realizando un papel importante, por ejemplo, en Canadá, Alberta AB-Edmonton, Calgary, Royal Roads British y Ontario Institute for Studies in Education, proponen su modelo en el uso de tecnología, en diseños metodológicos y en el trabajo colaborativo entre estudiantes y tutores. Así mismo, en Estados Unidos, se presenta el mismo enfoque en las Universidades de California, Berkeley, Global University Network for Innovation, Georgetown University, Central Florida, Rioja, Illinois de UrbanaChampaign y Carolina del Norte (Domínguez, 2008).

En Latinoamérica, se destaca, desde 1996, el Modelo Pedagógico Virtual del Instituto Tecnológico de Estudios Superiores de Monterrey de México (ITESM, 2008), sus cursos se desarrollan a través de conocimientos relevantes, significativos, actividades que promueven la colaboración, autogestión del aprendizaje por parte de los estudiantes, evaluación y retroalimentación continua del progreso de los mismos.

Chile, ha sido pionero en Suramérica en modelos pedagógicos virtuales, apoyados en el uso de computadoras y de redes. La Pontificia Universidad Católica de Chile adelanta un diseño de aprendizaje de desarrollo de empresas, por medio de tres enfoques: gestión, tecnología y educación, que permiten generar una dinámica encaminada a maximizar las competencias. En Argentina, las universidades Nacional de Quilmes, Nacional Tres de Febrero, Maimónides Online, Virtual del Nordeste, Virtual de Rosario y Universidad Virtual Juan Agustín Mazza, sustentan el diseño pedagógico virtual de desarrollo empresarial con apoyo de las TIC en estrategias didácticas en la producción, análisis, documentación y creatividad.

En Brasil, universidades como Nacional de Río Cuarto, Amazonía, Pontificia Católica de Campinas, Federal de Santa Catarina, São Paulo, Pontificia Universidad Católica de São Paulo, do Estado de Sao Paulo y Virtual Pública, basan su formación en el uso creativo y adecuado de recursos, con las exigencias empresariales del país (Angeloni, 2006).

En Colombia: la Universidad Nacional Agraria de la Selva fue una de las primeras en incorporar esta modalidad, mediante convenio con el ITESM. Su modelo pedagógico empresarial, se fundamenta en promover el liderazgo y la excelencia en la formación de profesionales, con un enfoque científico, tecnológico, humanístico y social, apoyado en plataformas. Igualmente, la Fundación Universitaria Católica del Norte, desde 1998, ofrece programas en la modalidad virtual, soportado en su propio software educativo.

La Universidad de la Empresa, en asocio con la UOC y luego con la Universidad Nacional de Quilmas, creó en 2000, un campus virtual para brindar estudios en Gestión del Conocimiento. Un año más tarde, siete instituciones colombianas formaron una alianza estratégica con la Red Universitaria Mutis (RUM) con ITESM, para asumir este modelo de educación virtual, integrada por las Universidades Autónoma de Bucaramanga (UNAB), Autónoma de Occidente (UAO), Tecnológica de Bolívar (UTB), Coruniversitaria de Ibagué, Autónoma de Manizales (UAM), Minuto de Dios y las Fundaciones Manuel Mejía y Suramericana.

La UNAB, caracteriza su modelo pedagógico, tecnológico y comunicativo, en el diseño de materiales e-learning desde la perspectiva de la gestión empresarial. El formato educativo y tecnológico de la UAO es similar al del ITESM con el respaldo del Instituto Latinoamericano para la Comunicación Educativa (ILCE), que se centra en el estudiante y el cual, requiere conocimientos básicos de cómputo, organización del tiempo y responsabilidad que asume para conformar su propia empresa.

El modelo e-learning de Uniminuto es parecido al de la Fundación Universitaria de Popayán y al de la Universidad Tecnológica de Pereira, se apoya en el Instituto de Educación Virtual y a Distancia con sus plataformas Manhattan y Cátedra, prioriza la responsabilidad, la dedicación, la autonomía del alumno, como agente activo de su proceso de aprendizaje. Con el mismo propósito, la Universidad Santiago de Cali promueve un enfoque de calidad, flexible, accesible y didáctico, basado en problemas y en la construcción de comunidades de conocimiento. 
De la misma forma, otro grupo de universidades colombianas han comenzado a trabajar sobre proyectos de educación virtual con énfasis empresarial, mediante una formación pedagógica dual y otras, a mediano plazo, emprenderán acciones incorporando las TIC, entre ellas: Antioquia, Los Andes, Nacional de Colombia, Sergio Arboleda, La Gran Colombia, Militar Nueva Granada, Escuela de Administración, Finanzas y Tecnología (EAFIT), Católica del Norte, Pontificia Javeriana, Pedagógica Nacional, Industrial de Santander, Pamplona, Valle, San Buenaventura, Santo Tomás y Universidad de Ciencias Aplicadas y Ambientales U.D.C.A.

De acuerdo a este estudio, 94\% de universidades presentan modelos pedagógicos virtuales de desarrollo empresarial con apoyo de herramientas tecnológicas en pregrado y postgrados; $57 \%$, están en exploración de sus diseños; $75 \%$, ingresa desde cualquier parte de la Institución y $84 \%$, cuenta con acceso a Internet por fibra óptica, poseen portales electrónicos e Intranets, lo que facilita la interacción entre la universidad y los empresarios.

En pedagogía y autoaprendizaje, $81 \%$, ofrece seminarios de inducción empresarial; $51 \%$, capacitación permanente; $42 \%$, tiene aulas virtuales; $26 \%$, cursos en línea soportados en equipos multimedia y $23 \%$, videoconferencia. En cuanto a programas ofrecidos bajo esta modalidad, se encuentran: Administración (20,3\%), Educación (18,7\%), Ciencias Sociales (10\%), Economía (9\%), Ingeniería (8\%), Tecnología (8,3\%), Ciencias Básicas (7\%), de la Salud $(6,2 \%)$ y otros $(4,5 \%)$, los cuales, funcionan con una plataforma enseñanza-aprendizaje diseñada según las necesidades de cada universidad (63\%) y otras con sitios Web comerciales (37\%).

De 57 modelos pedagógicos virtuales de Europa, se observaron en 20 de ellos, tendencias a conformar redes virtuales empresariales, con el fin de hacer parte del tejido mundial para la investigación y la educación entre entidades gubernamentales, empresas y otros sectores. Mientras que de 18 modelos pedagógicos en línea de Norteamérica y 48 en Latinoamérica, en 35 de ellos fundamentan un ambiente dinámico en Internet, como apoyo a la labor pedagógica, para facilitar mecanismos alternos de comunicación.

En síntesis, 44 de las universidades estudiadas disponen de un portal digital propio; $7 \%$ tiene servicios de hosting o arrendamiento de servidores; $41 \%$ posee aulas virtuales; $88 \%$ cursos en línea empresariales; $13 \%$ emplea video y audio conferencias; $73 \%$ biblioteca virtual; $91 \%$ comunicaciones virtuales y $12 \%$ tecnología WAP.

Se deduce de los resultados obtenidos en estudio del estado del arte propuesto, que se requiere de modelos y de metodologías virtuales de desarrollo empresarial que integre: educación-tecnología, docente-discente, enseñanza-aprendizaje, contenidos-competencias y comunicación-información, todas ellas enmarcadas en la implementación del uso de las TIC, como medio eficaz para afrontar las nuevas metas educativas y empresariales, es decir, un formato pedagógico que esté acorde con el desarrollo de sistemas educativos innovadores y que posibilite un currículo flexible adecuado para el desarrollo de las competencias empresariales.

Propuesta de un diseño pedagógico virtual de desarrollo empresarial con apoyo de las TIC: de acuerdo con los resultados arrojados, se considera que la mejor aproximación para el aprendizaje virtual y manejo de información empresarial que utiliza bases de datos es la Gestión de Conocimiento (GC), entendida como el proceso sistemático de detectar, seleccionar, organizar, filtrar, presentar y usar la información por parte de los participantes de un entorno, con el objeto de aprovechar, cooperativamente, los recursos de conocimiento basados en el capital intelectual propio de las organizaciones y orientado a potenciar las competencias de sus miembros (Figura 1).

El aprendiz, detecta las fuentes de acuerdo a sus capacidades cognitivas (modelos mentales, visión sistémica y otras), las selecciona y evalúa, mediante su pensamiento crítico, de acuerdo con los objetivos y los criterios trazados por el docente en cada una de las temáticas. El estudiante categoriza la información para determinar diferentes niveles de importancia y adaptarla para desenvolverse en nuevas situaciones comunicativas.

En este enfoque comunicativo, las estrategias se agrupan en: organizar y clasificar la información, a fin de generar nuevas ideas para ser discutidas colaborativamente: las almacena de forma estructurada y explicita en el modelo. Para tal objetivo, se debe realizar las siguientes etapas: Generar: crear ideas e identificar nueva documentación, páginas Web y libros. Buscar: en la medida en que no 


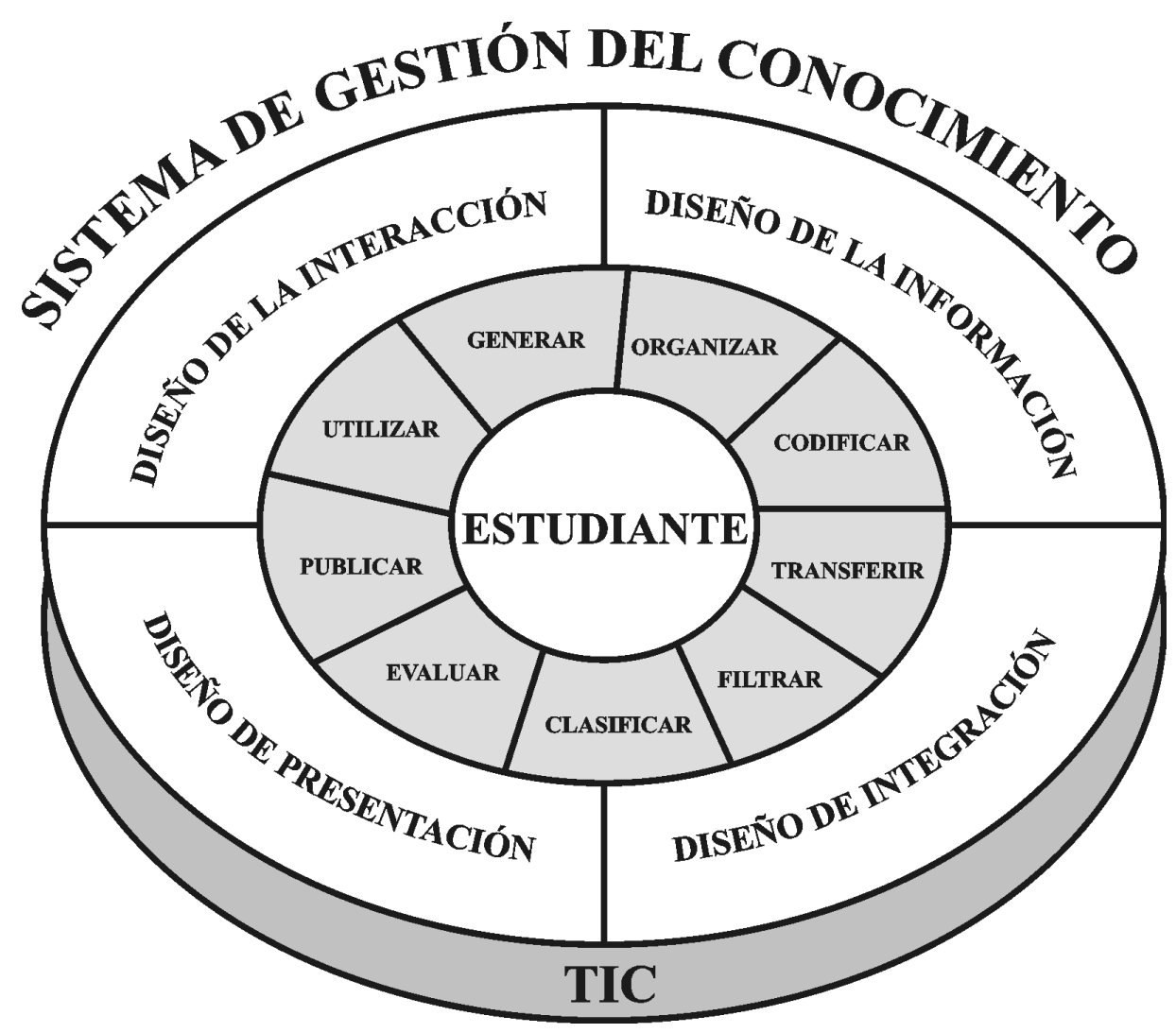

Figura 1. Diseño pedagógico virtual de desarrollo empresarial con apoyo de las TIC.

todos los alumnos accedan a la misma información será posible realizar trabajos de complementariedad, unos pueden hacerlo mediante audio y video y, otros, en Webs interactivas.

No basta con encontrar datos y presentarlos, deben ser transformados en información, recopilados, analizados, seleccionados, estructurados y organizados. Una vez elaborados y modificados en información para un propósito específico, deben tener la posibilidad de ser recuperados cuando sea necesario, para aplicarlos en la resolución de situaciones que se presenten en otros momentos. "Este proceso depende cada vez más del manejo de datos, información y conocimiento. Si no se intercambian saberes, no se crea riqueza" (Toffler, 2001).

Codificar: al tener la información seleccionada, se ingresa a su grupo o comunidad virtual en Internet, con un lenguaje común (palabras, diagramas, imágenes), a fin de construir conocimiento colaborativo, ayudado por interfaces y por herramientas de la Web. Transferir: establece el almacenamiento y apertura del conocimiento, a través de la red o sitios Web. Filtrar: una vez organizadas las fuentes de información, se contrastan, mediante consultas automatizadas en torno a motores de búsqueda, a portales o agentes inteligentes. El estudiante investiga, trabaja en equipo y empieza a crear habilidades, con el objeto de generar conocimiento.

Presentar: los resultados obtenidos del proceso de filtrado son expuestos a la comunidad, a través de un documento final, con el propósito que estos nuevos conocimientos sean reconstruidos y actualizados conjuntamente. Usar y difundir: luego, de construido grupalmente el nuevo saber, se publica en un portafolio digital, con el ánimo que sea utilizado para otras tareas.

Este diseño de desarrollo empresarial necesita de un Sistema de Gestión de Conocimiento (SGC), que no sólo almacena información en forma de 
noticias, sino que se va más allá; además, incorpora mecanismos de comunicación que permiten compartir el conocimiento. Igualmente, el empleo de las TIC, como enfoque educativo, facilita el aprendizaje y la utilización de un SGC, que acepte establecer la mayor cantidad de relaciones entre la información disponible y su clasificación a diferentes niveles. A partir de este soporte de conocimiento, se planeará el aprendizaje, a través de la acción, que se reflejará en la ejecución de tareas. En este contexto, el sistema interactuará con el aprendiz para motivarlo y le presentará la información personalizada y adaptada a sus preferencias.

En cuanto al conocimiento que se debe manejar, se requiere una modificación de los modos de aprender y enseñar. Esta transformación implica el paso de un modelo en el que el docente es el agente monopolizador del conocimiento y su representante autorizado, a otro en el que el alumno es llamado a la exploración individual y al autoaprendizaje relacionado con la realidad empresarial. En este tipo de patrones, el alumno construye las relaciones, descubre el proceso a medida que se involucra en él y es estimulado a trazar su propio recorrido (Piaget, 1999).

Para alcanzar este aprendizaje es necesario plantear estrategias de acción que no sean vistas como pesadas obligaciones sino como entretenidas opciones de aprendizaje. Así, se presentan los contenidos no como algo a estudiar, sino como elementos necesarios para obtener unos objetivos (Martínez, 2002), que se irán descubriendo, por medio de diversas pruebas, aprendiendo a avanzar por las diferentes fases mediante pruebas, errores, experiencias obtenidas en momentos previos y desarrollando habilidades que mejoran con la práctica.

A continuación, se mostrarán las fases que soportarán el desarrollo empresarial, las cuales, tienen en cuenta los principales procesos que intervienen en los sistemas interactivos: diseño de la información, diseño de la interacción, diseño de la presentación y diseño de la integración.

Diseño de la información: comprende la identificación de unidades y de procesos, división en subprocesos y la creación de mapas conceptuales. A partir de estos últimos, se asocian los elementos de conocimiento a cada uno de ellos para asignarle un problema modelo y cuestionamientos reales. Una vez organizados, se deben incluir en la Base de Conocimiento (BC).

Diseño de la interacción: para unir la estructura no lineal de la información y el aprendizaje activo por experiencia, se propone una forma de almacenar y acceder a los conocimientos de manera progresiva con: la introducción al entorno, establecimiento de preferencias de aprendizaje y de visualización, cuestionario general del curso, nivel inicial y temas del mismo. En lugar de asociar contenidos, se presentan pruebas que requieren del conocimiento a adquirir para ser realizadas.

El discente dispone de ayudas como: materiales básicos para la resolución de las tareas en diversos formatos, enlaces a temas relacionados, ejemplos de problemas, propuestas de otros compañeros para llegar a la solución, consejos de lo que debe y no debe hacer, a quién puede preguntar, entre otras inquietudes. De esta manera, el estudiante irá construyendo, a través de la práctica, su propio saber. En el momento en que llegue a la solución de una tarea, se le propondrán otras más complejas que precisa del conocimiento adquirido en la realización de la tarea anterior.

A fin de permitir la relación temática, se debe considerar la exposición de los mismos, evaluación, refuerzo y aclaración de conclusiones previas, retroalimentación, planteamiento de problemas, directorio electrónico de consulta, lecciones aprendidas, preguntas más frecuentes, cuaderno de evaluación y presentación de conclusiones, para reafirmar los conocimientos adquiridos. Estas ayudas que puede emplear el alumno, se estructurarán siguiendo las directrices de la GC, como: directorio de búsqueda, que incluirán documentos de diversos formatos: imágenes, vídeos, textos, bibliografía recomendada, listado de expertos en el tema.

Diseño de la presentación: contará con la introducción, que incluye la exposición del tema, objetivos de aprendizaje, estrategias que se aplicarán y su relevancia. En cuanto al problema, contendrá grado de dificultad, oportunidad, ayudas que se pueden generar y retroalimentación. Finalmente, el resumen, compuesto de conceptos claves, tipos de cuestionamientos resueltos y objetivos alcanzados. De acuerdo con lo anterior, se seleccionará las técnicas para hacer la tecnología transparente al usuario. De igual manera, se propone la utilización de un entorno Web que evita la instalación de 
programas específicos, a excepción de los necesarios, para el manejo de determinados formatos.

Diseño de la integración: para optimizar esta fase, se debe permitir la comunicación entre los elementos y el alumno. Se utilizan cinco tipos de estrategias, que facilitan el reparto de tareas entre los ordenadores locales y el servidor. Para tal fin, se contará con el monitor del discente, que trabaja localmente para controlar cambios en el estado del estudiante y generar un perfil del mismo, que servirá para personalizar la presentación de la información; el monitor de la base del conocimiento (BC) que interrelaciona remotamente, para controlar cambios; el estratega quien gestiona en el equipo local tácticas de aprendizaje, según preferencias y nivel del discente.

Validación del diseño pedagógico virtual de desarrollo empresarial con apoyo de las TIC: se aplicó el diseño en los dos cursos académicos referenciados, obteniendo los siguientes resultados: 13\%, destacó la interacción, el papel del docente y del discente en el proceso de enseñanza y aprendizaje; $88 \%$, consideró suficiente la capacitación sobre el modelo; $98 \%$, se conectó a internet entre 90 y 105 veces durante cada curso; 96\%, resaltó la diversidad de posibilidades que aportó el diseño pedagógico de desarrollo empresarial; $45 \%$, explicitó la actualidad de las informaciones y de encontrarse dentro de un entorno nuevo y $91 \%$, manifestó que el modelo, con apoyo de Internet, se convierta en canal de diferentes cursos académicos.

Se presentaron problemas por deficiencia de la infraestructura disponible, lentitud de acceso-transmisión de datos y pocos ordenadores conectados a la red. Sin embargo, $94 \%$ respondió que los medios y las tecnologías permitieron mayor interacción con los docentes, $80 \%$ tiene capacidad de usar las TIC, $12 \%$ presentó nivel medio de conocimiento, mientras que $5 \%$ tiene nivel bajo y 2,5\% nivel muy bajo. 95\% consideró que los contenidos fueron claros y didácticos respetando la propiedad intelectual y ponderaron la exigencia del docente lo que aseguró la calidad de cada asignatura y la optimización del aprendizaje, debido a su continua revisión y actualización.

Se destacó el material diseñado para la metodología: presentación del curso, guía de aprendizaje, contenidos, acciones de fundamentación: árbol conceptual, marco referencial Web, lecturas, navegación guiada, acciones de retroalimentación, autoevaluación conceptual, talleres de trabajo, aplicación y evaluación. 87\%, resaltó los contenidos desarrollados, su presentación, accesibilidad y funcionalidad de los enlaces, calidad, variedad y dinámica de las interacciones; $91 \%$, destacó que el contenido estuvo relacionado con el contexto actual, con cada curso académico y con las exigencias empresariales, gracias al aumento paulatino del volumen de documentación del curso disponible con sus respectivos vínculos.

Frente a la bibliografía propuesta, el 91\% expresó que la bibliografía fue suficiente como también la disponibilidad de espacios físicos y virtuales; $96 \%$, destacó al syllabus como dinámico y pertinente y $75 \%$, resaltó los foros virtuales. Una de las herramientas importantes fue el uso del correo electrónico del facilitador para retroalimentar el proceso del discente, herramienta que sirvió para enriquecer el aprendizaje. El 92\% enfatizó el cambio del papel del tutor y del aprendiz en el uso y apoyo virtual, creación de nuevos espacios de comunicación y su aplicación en contextos empresariales.

\section{CONCLUSIONES}

Los modelos pedagógicos virtuales de desarrollo empresarial que implementan las universidades estudiadas, se fundamentan en la enseñanzaaprendizaje, el alumno cumple papel activo en el proceso autodirigido, colaborativo y significativo apoyado por las TIC, a través de seguimiento, tutoría, actividades, estrategias didácticas y reflexión pedagógica, que se sustentan en siete elementos: colaboración asincrónica, disponibilidad de un calendario, tutoría, construcción de una comunidad, límite de discentes por grupo, calidad de materiales y espacios virtuales.

Lo expresado invita a las universidades a innovar sus tradicionales modelos de aprendizaje y tecnologías para llegar a la demanda de redes de convergencia mundial y regional, para facilitar el acceso a los servicios de información y comunicación, con el ánimo de establecer un nuevo modelo basado en el principio de movilidad, que permita integrar mapas de conocimiento visual, bancos de imágenes, herramientas multimedia, internet y tecnologías Web 2.0, para soportar el proceso de enseñanza-aprendizaje en sus diferentes modalidades. 
Las TIC facilitaron el trabajo colaborativo, porque los aprendices compartieron información, trabajaron con documentos conjuntos y tomaron decisiones para la solución de problemas, con ayuda de herramientas tecnológicas, con base en asignación de tareas, calendarios, convocatoria de reuniones, lluvia de ideas, mapas conceptuales y notas. Las tecnologías propiciaron nuevos espacios, oportunidades de cooperación y participación, que conllevaron a un aprendizaje cooperativo, mejorando la convivencia y el trabajo en equipo, lo que evidenció el progreso individual y colectivo de los discentes. Las estrategias pedagógicas, didácticas y metodológicas de aprendizaje fueron bien evaluadas.

Proponer este nuevo diseño pedagógico virtual de desarrollo empresarial con apoyo de las TIC contribuye a la reflexión de un proceso pedagógico que permite que los estudiantes aprendan sin desvincularse de sus problemas regionales, lo que representa un ejemplo de la interacción necesaria entre lo global y lo local, aspecto determinante para alcanzar la propia pertinencia de un nuevo modelo de enseñanza-aprendizaje virtual. Estos objetivos no serán alcanzados a menos que las universidades nacionales adopten toda la gama de TIC en la Web, que van desde la implantación de procesos de aprendizaje en aulas-laboratorios y la utilización eficiente de la enseñanza a distancia, hasta la producción de material didáctico orientado a las necesidades.

El diseño pedagógico virtual de desarrollo empresarial con apoyo de las TIC, basado en la gestión del conocimiento, permite tener en cuenta las interrelaciones existentes entre la información que se maneja y proporciona. A partir de esta propuesta, se dejan algunas preguntas que pueden orientar investigaciones similares en esta temática: la creación de un software gratuito en la red coptimizará los sistemas de información empresarial? Al aunar esfuerzos, las universidades y las empresas podrán proponer nuevos diseños pedagógicos en la Web 2.0, acorde con las necesidades del entorno?

\section{BIBLIOGRAFÍA}

ANGELONI, M. 2006. Organizações do Conhecimento - Infra-Estrutura, Pessoas e Tecnologías. Ed. Saraiva (São Paulo, Brasil). 123p.

ÁREA, M. 2005. Las tecnologías de la información y comunicación en el sistema escolar. Una revisión de las líneas de investigación. Rev. Electr. Invest. Eval. Educ. 11(1):1-15 Disponible en Internet desde: http://www.uv.es/RELIEVE/v11n1/ RELIEVEv11n1_1.pdf (con acceso 23/03/08).

BEDNAR, A., CUNNINGHAM, D., DUFFY, T., PERRY, J. 2007. Theory into practice: How do we link? In: Duffy, T.M.; Jonassen, D.H. (Eds.), Constructivism and the Technology of instruction: A conversation. Hillsdale, N.J.: Lawrence Erlbaum Associates. 97p.

CONCORD CONSORTIUM E- LEARNING. 2008. Model for Online Courses Disponible desde Internet en: http://www.concord.org/courses/cc_e-learning_ model.html (con acceso 05/03/08).

DENIS, Z. 1998. La formación del profesorado en tecnologías de la información en la educación. Ed. Anaya (Madrid, España). 45p.

DOMÍNGUEZ, G. 2008. Perspectiva de la Universidad en la Escena Contemporánea. Ed. Uladech (Chimbote, Perú). 11p.

EDUTEKA. 2008. Aprendizaje virtual. Rev. Fundación Gabriel Piedrahita Uribe (Colombia). 22(1):10-13.

FUNDACIÓN GABRIEL PIEDRAHITA URIBE. 2007. Modelo y metodología Gavilán para desarrollar la competencia para manejar información (CMI) Disponible desde Internet en: http:// www.ribiecol.org/index2.php?option $=\mathrm{com}$ docmanEtask $=$ doc_viewEgid $=27$ Eltemid $=1 \overline{5}$ (con acceso 08/30/08).

GUERRERO, R.; DÍAZ, P. 2007. Diseño de una herramienta para la creación de cursos virtuales. Ed. Teleduc (La Habana, Cuba). 85p.

INSTITUTO TECNOLÓGICO DE MONTERREY. 2008. Modelo de educación virtual. Disponible desde Internet en: www.ruv.itesm.mx (con acceso 07/08/08).

MARTÍNEZ, J. 2002. Contenidos en e-learning: el rey sin corona. Disponible desde Internet en: http:// www.uoc.edu/dt/20126/index.html (con acceso 07/11/08). 
MONEREO, C. 2007. Las estrategias de aprendizaje: preguntas básicas para la integración en la programación didáctica. Ed. Visor (Madrid, España). 162p.

PIAGET, J. 1999. De la pedagogía. Ed. Paidós (Buenos Aires, Argentina). 123p.

SAETTLER, P. 2005. The evolution of american educational technology. Englewood, CO: Libraries Unlimited, Inc. Teorías de Bloom y Gagñe. 234p.

SUENGAS, J., GONZÁLEZ, J. 2003. Estrategias metacognitivas. Aprender a aprender y aprender a pensar. Ed. Síntesis (Madrid, España). 27p.
TINKER, R. 2008. E-Learning Quality: The Concord Model for Learning from a Distance. NASSP Bulletin. 85(628):37-46.

TOFFLER, A. 2001. La Tercera Ola. Ed. Plaza y Janés (Barcelona, España). p.53.

UNIVERSIDAD OBERTA DE CATALUÑA. 2008. Modelo Pedagógico. Disponible desde Internet en: http:// www.uoc.edu/portal/castellano/ (con acceso 04/07/08).

YARZÁBAL, L. 2004. La transformación universitaria en víspera del tercer milenio, Volumen 6, Serie Políticas y Estrategias CRESALC/UNESCO. (Caracas, Venezuela).145p.

Recibido: Agosto 26 de 2008

Aceptado: Abril 23 de 2009 